\title{
Some Partial Results on Linial's Conjecture for Matching-Spine Digraphs*
}

\author{
Jadder Bismarck de Sousa Cruz ${ }^{1}$, Cândida Nunes da Silva ${ }^{2}$, Orlando Lee $^{1}$ \\ ${ }^{1}$ Institute of Computing, University of Campinas, Brazil \\ ${ }^{2}$ Department of Computing, Federal University of São Carlos, Brazil \\ bismarckeic.unicamp.br, candida@ufscar.br, leeeic.unicamp.br
}

\begin{abstract}
Let $k$ be a positive integer. A partial $k$-coloring of a digraph $D$ is a set $\mathcal{C}$ of $k$ disjoint stable sets and has weight defined as $\sum_{C \in \mathcal{C}}|C|$. An optimal $k$-coloring is a $k$-coloring of maximum weight. A path partition of a digraph $D$ is a set $\mathcal{P}$ of disjoint paths of $D$ that covers its vertex set and has $k$-norm defined as $\sum_{P \in \mathcal{P}} \min \{|P|, k\}$. A path partition $\mathcal{P}$ is $k$-optimal if it has minimum $k$ norm. A digraph $D$ is matching-spine if its vertex set can be partitioned into sets $X$ and $Y$, such that $D[X]$ has a Hamilton path and the arc set of $D[Y]$ is a matching. Linial (1981) conjectured that the $k$-norm of a $k$-optimal path partition of a digraph is at most the weight of an optimal partial $k$-coloring. We present some partial results on this conjecture for matching-spine digraphs.
\end{abstract}

\section{Introduction}

For a digraph $D$, let $V(D)$ denote its vertex set and let $A(D)$ denote its arc set. Given an arc $a=(u, v) \in A(D)$, we say that $u$ and $v$ are adjacent and also that $u$ is the tail and $v$ is the head of $a$. The set of neighbors of a vertex $u$ in $D$, denoted by $N(u)$, is the set of vertices that are adjacent to $u$. In this paper, we consider only digraphs without loops and parallel arcs. A path is a sequence of distinct vertices $P=\left(v_{1}, v_{2}, \ldots, v_{\ell}\right)$ such that $\left(v_{i}, v_{i+1}\right) \in A(D)$ for every $1 \leq i \leq \ell-1$. We denote by $V(P)$ the set of vertices of $P$ and by $\operatorname{ter}(P)$ the vertex $v_{\ell}$. We define the order of a path $P$, denoted by $|P|$, as its number of vertices. We denote by $P v_{i}$ the subpath $\left(v_{1}, v_{2}, \ldots, v_{i}\right)$ of $P$. A Hamilton path in $D$ is a path containing every vertex in $V(D)$. A cycle is a sequence of vertices $C=\left(v_{0}, v_{1}, \ldots, v_{\ell}\right)$ with $\ell \geq 2$ such that $\left(v_{i}, v_{i+1}\right) \in A(D)$ for every $0 \leq i \leq \ell-1$, and all vertices are distinct except $v_{0}$ and $v_{\ell}$ which coincide. We say that a digraph $D$ is acyclic if it does not contain a cycle. A digraph $D$ is transitive if whenever $(u, v) \in A(D)$ and $(v, w) \in A(D)$, then $(u, w) \in A(D)$ as well.

A path partition $\mathcal{P}$ of a digraph $D$ is a set of disjoint paths that cover $V(D)$. A path partition $\mathcal{P}$ of $D$ is optimal if it has minimum cardinality, and we denote its cardinality by $\pi(D)$. Given a positive integer $k$, the $k$-norm of a path partition $\mathcal{P}$ of $D$, denoted by $|\mathcal{P}|_{k}$, is defined as $\sum_{P \in \mathcal{P}} \min \{|P|, k\}$. A path partition of $D$ with minimum $k$-norm is called $k$-optimal, and we denote its $k$-norm by $\pi_{k}(D)$. Note that $\pi(D)=\pi_{1}(D)$.

A stable set $S$ of a digraph $D$ is a subset of pairwise non-adjacent vertices. We denote by $\alpha(D)$ the cardinality of a maximum stable set of $D$. Given a positive integer $k$, a partial $k$-coloring $\mathcal{C}$ of $D$ is a set of $k$ (possibly empty) disjoint stable sets. The weight

\footnotetext{
*The first author was supported by Conselho Nacional de Desenvolvimento Científico e Tecnológico - Brasil (CNPq) and by the Coordenação de Aperfeiçoamento de Pessoal de Nível Superior - Brasil (CAPES) - Finance Code 001. The third author was supported by CNPq Proc. 303766/2018-2, CNPq Proc 425340/2016-3 and FAPESP Proc. 2015/11937-9.
} 
of a partial $k$-coloring $\mathcal{C}$, denoted by $\|\mathcal{C}\|$, is defined as $\sum_{C \in \mathcal{C}}|C|$. A partial $k$-coloring $\mathcal{C}$ is optimal if it has maximum weight, and we denote its weight by $\alpha_{k}(D)$. Note that $\alpha(D)=\alpha_{1}(D)$.

[Dilworth 1950] proved that the equality $\pi(D)=\alpha(D)$ holds when $D$ is a transitive acyclic digraph. [Gallai and Milgram 1960] generalized Dilworth's Theorem to arbitrary digraphs by relaxing the equality and establishing that $\pi(D) \leq \alpha(D)$ for every digraph $D$. [Greene and Kleitman 1976] generalized Dilworth's Theorem in a different way by showing that $\pi_{k}(D)=\alpha_{k}(D)$ for every transitive acyclic digraph $D$ and every positive integer $k$. [Linial 1981] conjectured that Greene-Kleitman's Theorem can be generalized to arbitrary digraphs by relaxing the equality in the same way that Gallai-Milgram's result extends Dilworth's Theorem.

Conjecture 1 (Linial's Conjecture). Inequality $\pi_{k}(D) \leq \alpha_{k}(D)$ holds for every digraph $D$ and every positive integer $k$.

Some particular cases of Conjecture 1 are solved such as $k=1$ (Gallai-Milgram's Theorem itself) and $k=2$ [Berger and Hartman 2008]. It also holds for some particular classes of digraphs ([Linial 1981, Berge 1982]). The following two recent results on Linial's Conjecture are relevant to this paper. A digraph $D$ is arc-spine if there is a partition $\{X, Y\}$ of $V(D)$ such that $D[X]$ has a Hamilton path and $D[Y]$ contains at most one arc; in particular, when $D[Y]$ is a stable set, we say $D$ is spine. [Sambinelli et al. 2017] proved Linial's Conjecture for spine digraphs and [Yoshimura et al. 2019] extended such result for arc-spine digraphs. This paper is a direct follow-up of the latter.

A digraph $D$ is matching-spine if there is a partition $\{X, Y\}$ of $V(D)$ such that $D[X]$ has a Hamilton path and the arc set of $D[Y]$ is a matching; clearly a superclass of arc-spine digraphs. We use the notation $D[X, Y]$ to indicate a partition with this property. In this paper we present partial results on Conjecture 1 for matching-spine digraphs.

\section{Main results}

Let $D[X, Y]$ be a matching-spine digraph, let $P$ be a Hamilton path of $D[X]$ and let $k \geq 2$ be an integer. The canonical path partition $\mathcal{P}$ of $D$ is the path partition consisting of $P$ together with all maximal paths of $D[Y]$; clearly $\pi_{k}(D) \leq|\mathcal{P}|_{k}=\min \{|X|, k\}+|Y|$. Let $Y^{+}$and $Y^{-}$be the subsets of vertices in $Y$ which are, respectively, tail and head of some arc in $D[Y]$ and let $Y^{0}=Y \backslash\left(Y^{+} \cup Y^{-}\right)$. A canonical partial $k$-coloring $\mathcal{C}$ is a partial $k$-coloring consisting of stable sets $Y^{0} \cup Y^{-}, Y^{+}$and $\min \{k-2,|X|\}$ singletons of $X$; clearly $\alpha_{k}(D) \geq\|\mathcal{C}\|=|Y|+\min \{k-2,|X|\}$. Note that there is a gap of two to reach our desired inequality.

A matching-spine digraph $D[X, Y]$ is $k$-tight if $|X| \geq k$ and for every $S \subseteq X$, with $|S|=k$, one of the following holds: (1) there is a vertex $y \in Y$ such that $S \subseteq N(y)$, or (2) there is an arc $\left(y^{+}, y^{-}\right) \in A(D[Y])$ and there is a vertex $s \in S$ such that $S-\{s\} \subseteq$ $N\left(y^{+}\right) \cap N\left(y^{-}\right)$and $s \notin N\left(y^{+}\right) \cup N\left(y^{-}\right)$. An alternative way to state condition (2) is the following: $s$ is the unique non-neighbor of $y^{+}$and $y^{-}$in $S$. When $D[X, Y]$ is not $k$-tight it is $k$-loose. The motivation for splitting the class of matching spine digraphs into $k$-loose and $k$-tight is the fact that it is easy to obtain a partial $k$-coloring with weight $\min \{|X|, k\}+|Y|$ for the $k$-loose ones. This implies the following.

Proposition 2. Let $k \geq 2$ be an integer and let $D[X, Y]$ be a $k$-loose matching-spine digraph. Then $\pi_{k}(D) \leq \alpha_{k}(D)$. 
Henceforth we assume that $D$ is $k$-tight. Let $P=\left(x_{1}, \ldots, x_{\ell}\right)$ be a Hamilton path of $D[X]$. We say that $P$ has a zigzag if one of the structures illustrated in Figure 1 exists. In Figure 1, $y$ denotes some vertex in $Y,\left(y^{+}, y^{-}\right)$some arc in $D[Y]$ and $i$ is some index such that $1 \leq i<\ell$. If $P$ has a zigzag there is a path $P^{\prime}$ of order $|X|+1$ and it is easy to show that $\pi_{k}(D) \leq|Y|+k-1$. If $P$ has no zigzag, then we say that $P$ is zigzag-free.

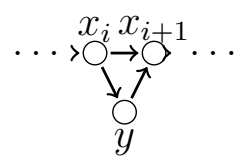

(a)

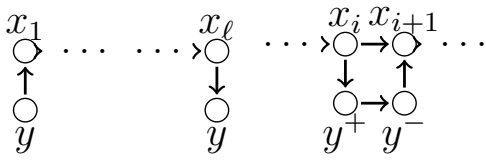

(b)

(c)

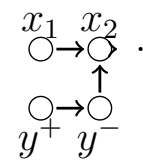

(e)

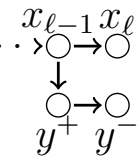

(f)

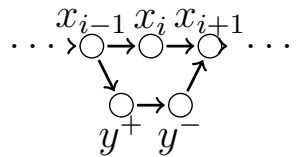

(g)

Figure 1. Types of zigzags of a path $P$.

Our main contribution in this paper is to show that $\pi_{k}(D) \leq|Y|+k-1$ for every $k$-tight matching-spine digraph $D$ (Theorem 4). To do so, we need Lemma 3 . We present just a sketch of the proof for the sake of conciseness.

Lemma 3. Let $k$ be a positive integer, let $D[X, Y]$ be a $k$-tight matching-spine digraph and let $P=\left(x_{1}, x_{2}, \ldots, x_{\ell}\right)$ be a zigzag-free Hamilton path of $D[X]$. Then, there are paths $P_{1}$ and $P_{2}$ such that: (i) $V\left(P_{1}\right) \cap V\left(P_{2}\right)=\emptyset$, (ii) $\left|P_{1}\right|+\left|P_{2}\right|=|X|+k+1$, and (iii) $\operatorname{ter}\left(P_{1}\right) \cup \operatorname{ter}\left(P_{2}\right)=\left\{x_{\ell}, y\right\}$, for some vertex $y \in Y$.

Sketch of the proof. We may assume that $Y$ is not a stable set, otherwise the result follows by [Sambinelli et al. 2017, Lemma 3]. The proof is by induction on $k$. The base case is $k \in\{1,2\}$. When $k=1$ take $P_{1}=P$ and $P_{2}$ as some arc in $D[Y]$ and the result follows. Surprisingly, the case $k=2$ is the longest part of the proof, requiring the analysis of many cases and is omitted. In the inductive step, we choose a vertex $x_{i} \in X$ such that $\left(x_{i}, y_{i}\right) \in A(D)$, for some $y_{i} \in Y$, and $i$ is maximum with this property. Since $P$ is zigzag-free, $x_{i+1}$ exists and $x_{i+1} \notin N\left(y_{i}\right)$. Let $Y^{\prime}=N\left(x_{i+1}\right) \cap Y$. By the choice of $i$, it follows that $\left(y^{\prime}, x_{i+1}\right) \in A(D)$, for every vertex $y^{\prime} \in Y^{\prime}$. Let $P^{\prime}=P x_{i}$ and let $X^{\prime}=V\left(P^{\prime}\right)$. We consider two cases. In case 1 , we assume that there is no $y \in Y^{+}$such that $\left(x_{i}, y\right) \in A(D)$. Let $Y^{\prime \prime}$ be the subset of vertices in $Y^{+}$which have no neighbor in $Y^{\prime}$. Let $D^{\prime}=D\left[X^{\prime} \cup Y^{\prime} \cup Y^{\prime \prime}\right]$. We can prove that $D^{\prime}$ is a $(k-1)$-tight matching-spine digraph and $P^{\prime}$ is zigzag-free. In case 2 , we assume that there is some $y \in Y^{+}$such that $\left(x_{i}, y\right) \in A(D)$. In this case we define $Y^{\prime \prime}$ as follows. A vertex $y^{+} \in Y^{+}$belongs to $Y^{\prime \prime}$ if there is an arc $\left(y^{+}, y^{-}\right) \in A(D[Y])$ such that $y^{+}, y^{-} \in N\left(x_{i+2}\right)$ and $y^{-} \notin Y^{\prime}$. Let $D^{\prime}=D\left[X^{\prime} \cup Y^{\prime} \cup Y^{\prime \prime}\right]$. We can prove that $D^{\prime}$ is a $(k-2)$-tight matching-spine digraph and $P^{\prime}$ is zigzag-free. In either case, we may apply the induction hypothesis to $D^{\prime}$ and $P^{\prime}$ to obtain $P_{1}^{\prime}$ and $P_{2}^{\prime}$ satisfying conditions (i)-(iii). We can extend $P_{1}^{\prime}$ and $P_{2}^{\prime}$ to paths $P_{1}$ and $P_{2}$ of $D$ satisfying conditions (i)-(iii) as desired.

Theorem 4. Let $k \geq 2$ be an integer and let $D[X, Y]$ be a $k$-tight matching-spine digraph. Then, $\pi_{k}(D) \leq|Y|+k-1$.

Proof. Let $P$ be a Hamilton path of $D[X]$. If $P$ has a zigzag, then $\pi_{k}(D) \leq|Y|+k-1$ and the result follows. So, we can assume that $P$ is zigzag-free. By Lemma 3 , there are paths $P_{1}$ and $P_{2}$ such that $\left|P_{1}\right|+\left|P_{2}\right|=|X|+k+1$. Let $\mathcal{P}$ be a path partition consisting of $P_{1}, P_{2}$ and all trivial paths in $V(D)-\left(V\left(P_{1}\right) \cup V\left(P_{2}\right)\right)$. Then, $|\mathcal{P}|_{k}=\min \left\{\left|P_{1}\right|, k\right\}+$ $\min \left\{\left|P_{2}\right|, k\right\}+|V(D)|-\left|P_{1}\right|-\left|P_{2}\right| \leq k+k+|X|+|Y|-(|X|+k+1)=|Y|+k-1$. 
By Theorem 4, $\pi_{k}(D) \leq|Y|+k-1$ for every $k$-tight matching-spine digraph $D$. Since $\alpha_{k}(D) \geq|Y|+k-2$, we now have a gap of one to reach the inequality of Linial's Conjecture. In an attempt to close this gap, we introduce a subclass of $k$-tight matching-spine digraphs. A matching-spine digraph $D$ is $k$-strongly-tight if $X$ is a clique of size at least $k$ and for every $T \subseteq X$ of size $k-1$ there is an $\operatorname{arc}\left(y^{+}, y^{-}\right) \in A(D[Y])$ such that $T \subseteq\left(N\left(y^{+}\right) \cap N\left(y^{-}\right)\right)$. We can show that if $D$ is a matching-spine digraph which is not $k$-strongly-tight, then $\alpha_{k}(D) \geq|Y|+\min \{k-1,|X|\}$, which leads to the following theorem.

Theorem 5. Let $k \geq 2$ be an integer and let $D[X, Y]$ be a matching-spine digraph which is not $k$-strongly-tight. Then, $\pi_{k}(D) \leq \alpha_{k}(D)$.

\section{Conclusion}

We have proved Linial's Conjecture for matching-spine digraphs which are not $k$ strongly-tight (Theorems 4 and 5). This result relies on Lemma 3, whose proof is nontrivial and is the main contribution of this paper. Only the proof for $k$-strongly-tight matching-spine digraphs is missing in order to settle Linial's Conjecture for matching spine digraphs. We believe that inequality $\pi_{k}(D) \leq|Y|+k-2$ holds for every $k$ strongly-tight matching-spine digraph. We have already proved the result for some cases that support this claim: for $k=2$ and for $|X|=k+1$.

\section{References}

Berge, C. (1982). k-Optimal partitions of a directed graph. European Journal of Combinatorics, 3(2):97-101.

Berger, E. and Hartman, I. (2008). Proof of Berge's strong path partition conjecture for $k=2$. European Journal of Combinatorics, 29(1):179-192.

Dilworth, R. (1950). A decomposition theorem for partially ordered sets. Annals of Mathematics, pages 161-166.

Gallai, T. and Milgram, A. (1960). Verallgemeinerung eines graphentheoretischen satzes von Rédei. Acta Sc. Math, 21:181-186.

Greene, C. and Kleitman, D. (1976). The structure of Sperner $k$-families. Journal of Combinatorial Theory, Series A, 20(1):41-68.

Linial, N. (1981). Extending the Greene-Kleitman theorem to directed graphs. Journal of Combinatorial Theory, Series A, 30(3):331-334.

Sambinelli, M., Nunes da Silva, C., and Lee, O. (2017). On Linial's conjecture for spine digraphs. Discrete Mathematics, 340(5):851-854.

Yoshimura, L., Sambinelli, M., Nunes da Silva, C., and Lee, O. (2019). Linial's conjecture for arc-spine digraphs. Electronic Notes in Theoretical Computer Science, 346:735746. 